\title{
Low Cost Air Quality Monitoring System Using LoRa Communication Technology
}

\author{
Mohammad Fadhli ${ }^{1, *}$ Asriyadi Asriyadi ${ }^{1}$, Lindawati Lindawati ${ }^{1}$, Irma Salamah ${ }^{1}$, \\ Gita Affrylia ${ }^{1}$, Michelle Valerie ${ }^{1}$, Andi Ramadhan ${ }^{1}$ \\ ${ }^{1}$ Politeknik Negeri Sriwijaya \\ *Corresponding author. Email: mohammad.fadhli@polsri.ac.id
}

\begin{abstract}
This paper proposes a low cost air quality monitoring system using LoRa-based communication technology. The proposed system consists of several sensor nodes and a gateway. Sensor nodes read pollutant levels in the air using the MQ-7 sensor for CO gas, MQ-135 for CO2 gas, GP2Y1010 for Particulates Matter (PM) and the DHT-22 sensor to read air temperature and humidity. Arduino UNO R3 is used as the data processing centre. Sensor data is sent to the gateway using the LoRa RF96 module. The gateway forwards the sensor data received to the Thingspeak server using NodeMCU $\mathrm{V} 3$, and users can access it via the internet network. The test results show that the designed system could read the levels of pollutants in the air and sent them to the Thingspeak server. Indoor-placed gateway could receive data from outdoorlocated sensor node with a $100 \%$ success rate up to a distance of 32 meters. While at a distance of 50 meters, the success rate of data reception by the gateway was $99.17 \%$ and at a distance of 70 meters was $78.3 \%$.
\end{abstract}

Keywords: Air Quality, LoRa, Arduino.

\section{INTRODUCTION}

Air pollution is one of the global problems that require attention from various parties. Air pollution occurs when various pollutants such as Carbon Monoxide (CO), Carbon Dioxide (CO2), Particulates Matter (PM) and various other harmful substances contaminating the air. These harmful substances come from various sources, one of which comes from the combustion of motor vehicles. In most developing countries, almost $70 \%$ to $80 \%$ of air pollution comes from old vehicles' emissions with poor engine maintenance [1].

Air pollution harms human health. Paper [2] states that exposure to $\mathrm{CO}$ gas in the air can cause asphyxia, leading to death if exposed to high concentrations. While in the article [3] stated that exposure to $\mathrm{CO} 2$ gas in the air can cause inflammation, decreased cognitive abilities, bone demineralization, kidney calcification, oxidative stress and endothelial dysfunction. PM are also pollutants that contribute to public health problems, where $7.6 \%$ of the global mortality rate is caused by PM with a diameter of less than $2.5 \mathrm{~m}\left(\mathrm{PM}_{2.5}\right)$ [4].

Several studies have designed air quality monitoring systems to prevent the negative impacts of air pollution.
Some of these systems utilize telecommunications technology. Studies [5]-[7] designed an air quality monitoring system by utilizing Internet of Things (IoT) technology. The proposed system consists of sensor nodes that use various sensors to detect the exposure of pollutants in the air, such as $\mathrm{CO}, \mathrm{O}_{2}$, temperature, and other harmful substances. The sensor readings are sent to the server via an internet connection. These systems can be used to monitor air quality from anywhere and anytime. However, this will be difficult if there is no internet connection at the sensor node location.

Other studies created air quality monitoring systems using ZigBee-based Wireless Sensor Network (WSN) technology, such as in studies [8]-[10]. ZigBee module allows multiple sensor nodes to communicate directly with the coordinator node, reducing the number of internet connections required. However, the ZigBee module has limitations regarding the communication distance between transmitter and receiver, about 25 meters for indoor locations and 60 meters for outdoor locations [11].

Study [12] designed an air quality monitoring system using MQ-7, MQ-135 and MQ-136 sensors to detect pollutants, Raspberry Pi as a data processing centre and 
LoRA module as a communication platform. The use of the LoRa module can overcome the problem of limited wireless communication distance between nodes. In this study [12], sensor nodes can communicate up to 400 meters in NLOS conditions and 1700 meters in LOS conditions.

This paper proposes an air quality monitoring system using MQ-135 sensors to detect CO2, MQ-7 to detect CO, GP2Y1010 to detect PM, and DHT-22 to read air temperature and humidity. All sensors are connected to the Arduino Uno R3, forming a sensor node. Arduino Uno is considered more efficient because it can be obtained at a lower price and has analogue I/O pins needed to read data from the sensor. Sensor node sends data to the gateway using LoRa module to be forwarded to the server using NodeMCU to access the sensor readings via the internet. The design of this system can connect many sensor nodes to one gateway. Hence, this system only requires an internet connection on the gateway side.

\section{SYSTEM DESIGN}

The air quality monitoring system proposed in this paper consists of several sensor nodes and one gateway. Sensor nodes act as readers of pollutant exposure in various locations. The types of pollutants observed are $\mathrm{CO}, \mathrm{CO} 2, \mathrm{PM}$, and reading the temperature and humidity of the air. Data from each sensor node are sent to the gateway using the LoRa module and then forwarded to the Thingspeak server to be accessed via the internet network. Figure 1 shows the overall system block diagram.

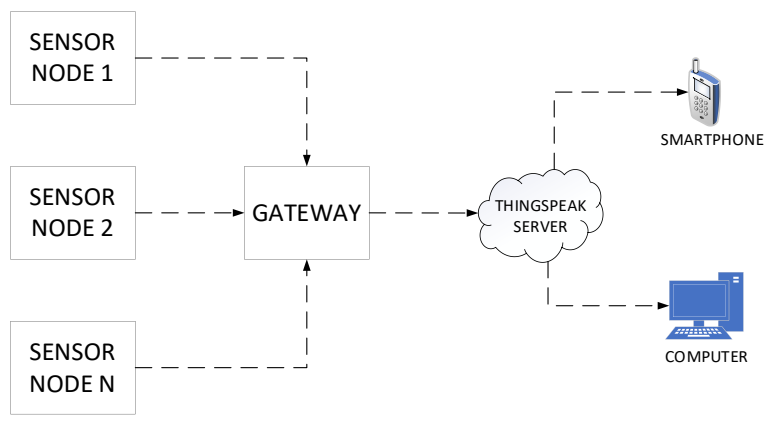

Figure 1 Overall System Block Diagram

\subsection{Sensor Node}

The sensor node consists of a sensor, a microcontroller, a communication module, and a realtime clock (RTC). Four sensors are used, namely the MQ-135 sensor to detect CO2, GP2Y1010 to detect PM, MQ-7 to detect CO, and DHT-22 to read air temperature and humidity. The microcontroller used is Arduino Uno $\mathrm{R} 3$ which is used to process sensor readings so that the pollutant content can be read in ppm for $\mathrm{CO} 2$ and $\mathrm{CO}$ gases, $\mathrm{PM}$ in $\mu \mathrm{g} / \mathrm{m} 3$, temperature in ${ }^{\circ} \mathrm{C}$ and humidity in percentage.
The proposed system uses LoRa RF96 as a communication medium between the sensor node and the gateway. The LoRa module aims to build a communication system with long distances and low power consumption. Each sensor node is equipped with an RTC DS3231, used as a data transmission timer to prevent data collisions between nodes. Thus each sensor node will only send data at the specified time, which is different for each sensor node.

Each sensor node is assigned an address number. The sensor node address is an integer starting from 1 and so on. The sensor node sends sensor data along with this address. The existence of this address number allows the gateway to identify the source of the data sender.

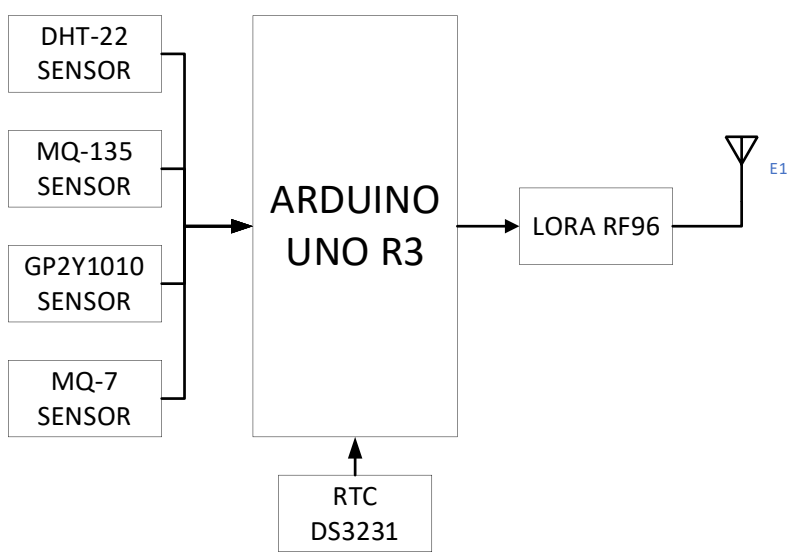

Figure 2 Sensor Node Block Diagram

\subsection{Gateway}

The gateway receives data from the sensor nodes and forwards it to the Thingspeak server. The gateway consists of a NodeMCU V3 and a LoRa RF96 module. NodeMCU V3 is a microcontroller board connected to a Wi-Fi network via the ESP8266 module, making it suitable for forwarding data to the internet network. Figure 3 shows the block diagram of the gateway.

The gateway first identifies the source of the data sender based on the address number contained in the received packet. The received sensor data is forwarded to the Thingspeak server and written to the field corresponding to the address number of the sending node. So data from different sensor nodes can be written in different fields. The sensor data on the Thingspeak server can be accessed online via computers and smartphones.

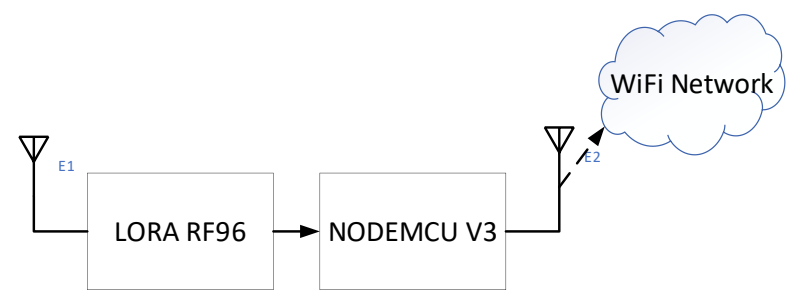

Figure 3 Gateway Block Diagram 


\subsection{LoRa Set Up}

The use of LoRa as a communication medium in Indonesia must comply with the Ministry of Information and Communication regulations. The regulations include the frequency used in the $920-923 \mathrm{MHz}$ range [13]. In addition, sending data packets using LoRa must also meet the duty cycle rules. The duty cycle compares the packet time on-air with the delay between packets sent by the node [14]. For the territory of Indonesia, the permitted duty cycle limit is $1 \%$. Packet time on-air can be calculated using the following equation [15]:

$\mathrm{T}_{\text {packet }}=\mathrm{T}_{\text {preamble }}+\mathrm{T}_{\text {payload }}$

$T_{\text {preamble }}=\left(n_{\text {preamble }}+4.25\right) T_{s}$

$T_{s}=1 / R_{s}$

$R_{s}=B W / 2^{S F}$

$T_{\text {payload }}=P L S y m b \times T_{S}$

$$
\begin{aligned}
& P L S y m b=8+ \\
& \max \left(\operatorname{ceil}\left(\frac{8 P L-4 S F+28+16 C R C-20 H}{4(S F-2 D E)}\right)(C R+\right. \\
& 4), 0)
\end{aligned}
$$

In equation (1), $\mathrm{T}_{\text {packet }}$ is the value of the time on air of the package, $\mathrm{T}_{\text {preamble }}$ is the duration of opening, and $\mathrm{T}_{\text {payload }}$ is the duration of the payload. While in equation (2), $n_{\text {preamble }}$ is the preamble length, and $T_{s}$ represents the symbol duration. In equation (3), $R_{S}$ is the symbol rate. In equation (4), BW represents bandwidth, and SF is spreading factor. In equation (5), PLSymb is the number of symbols carried in the payload, which can be calculated using equation (6). In equation (6), the PL represents the number of bytes in the payload. H indicates whether there is a header in each packet. This value is 0 if there is a header and 1 if there is no header. DE is 1 if uses the optimization system at low data rate conditions and 0 if it is not used. CR shows the value of the code rate.

Each sensor data is sent in an integer data type measuring 16 bits. There are five sensor parameters in this system: temperature, humidity, $\mathrm{CO}, \mathrm{CO} 2$, and PM. The total sensor data sent is 80 bits. In addition, the node address number is also sent using a 16-bit integer data type. Thus the total payload sent is 96 bits in size. This system was tested using spreading factor 7 , bandwidth $125 \mathrm{kHz}$, code rate 1, preamble length 8 , and without CRC. Based on these parameters, the packet's time on-air value is $41.22 \mathrm{~ms}$. Thus the allowed duty cycle is 4 seconds, meaning that data packets from each sensor node can be sent at the fastest every 4 seconds.

\section{RESULT AND DISCUSSION}

\subsection{Pollutant Exposure Measurement}

Testing the air quality monitoring system was carried out in the Sriwijaya State Polytechnic campus. Data collected is in the form of readings of $\mathrm{CO}$ and $\mathrm{CO}_{2}$ gas exposure in ppm units, PM exposure in $\mu \mathrm{g} / \mathrm{m} 3$, temperature in ${ }^{\circ} \mathrm{C}$ and the percentage of air humidity. Sensor node was placed in the corridor on the 3rd floor of the Electrical Engineering Laboratory of Sriwijaya State Polytechnic, which facing the parking lot. Gateway was placed in the laboratory building to make it easier to connect to a WiFi network. Sensor data retrieval was carried out from 09:30 to 16:00 WIB with packet delivery intervals from sensor node to gateway every 1 minute.

The results of temperature, humidity, $\mathrm{CO}, \mathrm{CO} 2$ and PM readings can be seen in Figures 4, 5, 6, 7 and 8. The highest temperature was 32 Celsius which occurred from 09:12 to 09:27. At this time, the temperature rose because the sun was rising from the east, where the sensor node was also facing east so that it got direct sunlight. Meanwhile, the temperature tended to be lower at the following times because the weather conditions were cloudy. Based on Figure 5, the highest humidity occurred in the morning at 08:30, which was $91 \%$. Meanwhile, the lowest humidity of $64 \%$ occurred at $13: 12$ to $13: 13$ and at $13: 16$ to 13.18 .

Figures 6 and 7 show the results of measurements of $\mathrm{CO}$ and $\mathrm{CO} 2$ gas exposure in the air. The lowest $\mathrm{CO}$ levels occurred several times at $20 \mathrm{ppm}$. Meanwhile, the lowest $\mathrm{CO} 2$ level of $333 \mathrm{ppm}$ occurred at 09:10 and 09.13. Peak CO levels occurred in the range of 15:22$15: 24$ at $29 \mathrm{ppm}$, and the highest $\mathrm{CO} 2$ gas exposure occurred at 15:24 at $721 \mathrm{ppm}$. The increase in $\mathrm{CO}$ and $\mathrm{CO} 2$ exposure could be caused by the increasing number of vehicles that pass through the sensor nodes. Figure 8 shows the PM values were read from 08:30 to 16:00. The smallest value occurred at $12: 42$ at $18 \mathrm{ppm}$, and the largest PM value occurred at 13:24 at $41 \mathrm{ppm}$.

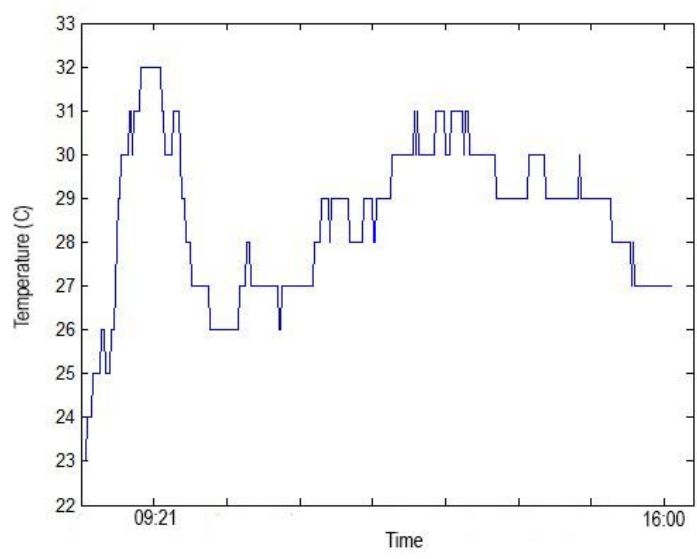

Figure 4 Temperature Measurement Results 


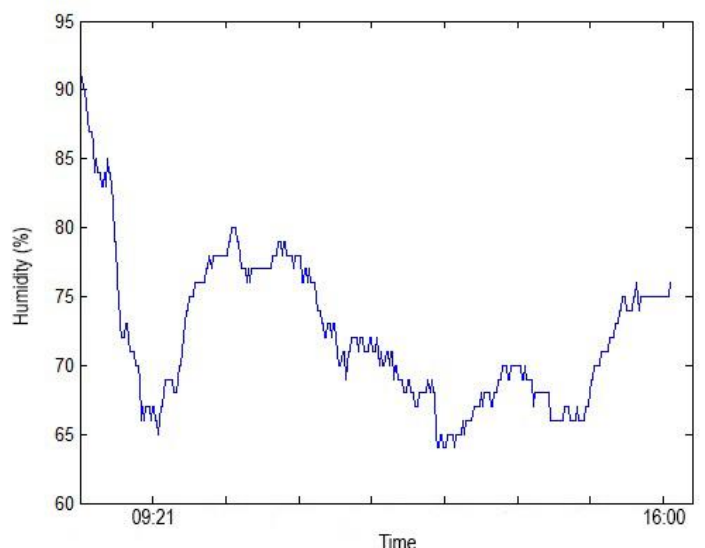

Figure 5 Humiduty Measurement Results

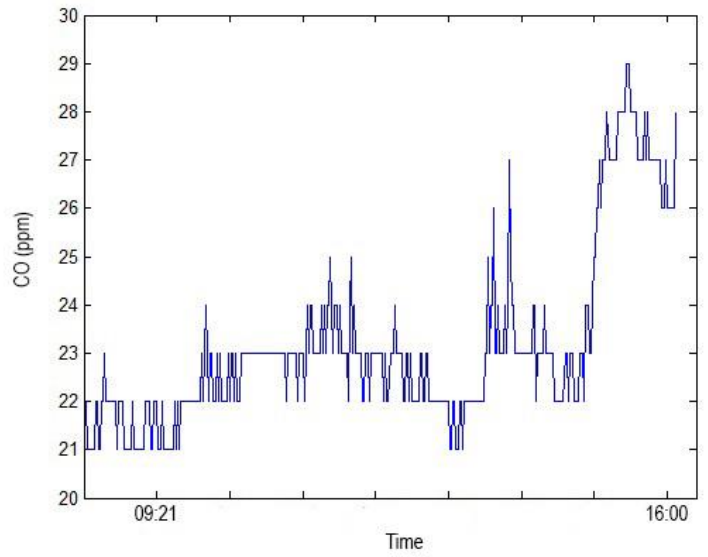

Figure 6 CO Measurement Results

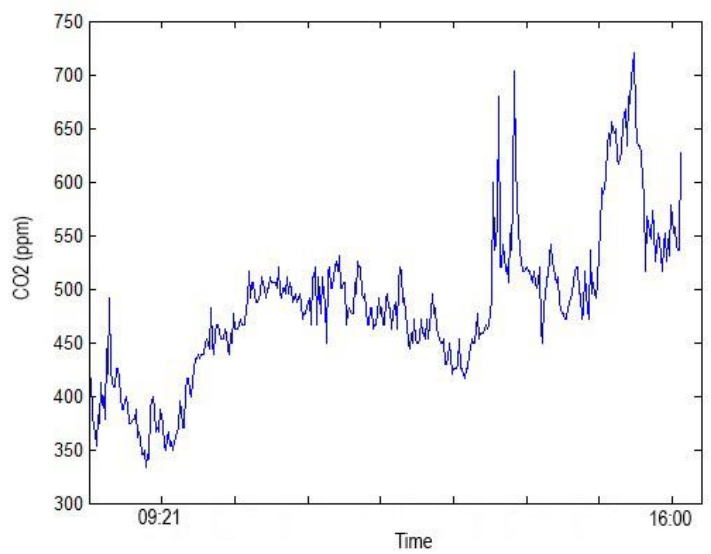

Figure $7 \mathrm{CO}_{2}$ Measurement Results

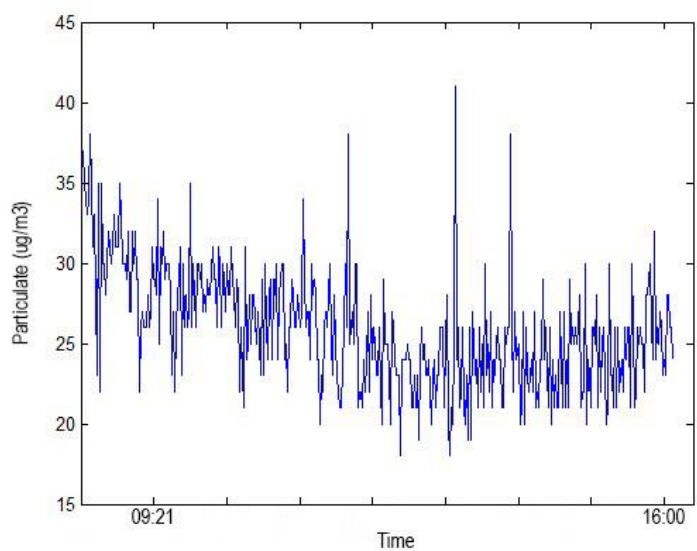

Figure 8 PM Measurement Results

\subsection{LoRa Communication Testing}

This study also tested the maximum communication distance between sensor node and gateway to determine the maximum performance limit that this system can carry out. Testing the communication range of sensor node and gateway was done by placing the sensor node in the same place as pollutant exposure measurement. Gateway was placed in three different positions in the building, namely at a distance of 18 meters, 32 meters, 50 meters and 69 meters from the sensor node location. The location of the measurement point can be seen in Figure 9. In Figure 9, Tx indicates the position of a sensor node, and another point indicates the gateway's position at various distances measured from the sensor node position. The collected data was the percentage of the number of packets successfully received by the gateway from 120 packets sent for each measurement point.

The results of testing the communication distance of sensor and gateway nodes can be seen in Table 1. Table 1 shows the number of packets that were successfully received without errors, the number of packets received with errors and the number of lost packets. At a distance of 18 meters and 32 meters, all packets sent could be received by the gateway without error. While at a distance of 50 meters there are 119 packets were successfully received. At a distance of 70 meters, only 94 packets were successfully received.

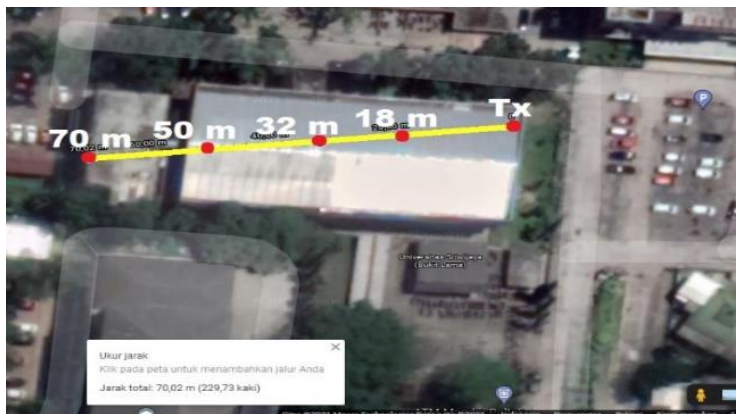

Figure 9 Location of Communication Distance Measurement Points 
Table 1. Communication Distance Measurement Results

\begin{tabular}{|c|c|c|c|c|}
\hline $\begin{array}{c}\text { Distance } \\
\text { (Meter) }\end{array}$ & $\begin{array}{c}\text { Sent } \\
\text { Packets }\end{array}$ & $\begin{array}{c}\text { Received } \\
\text { Packets }\end{array}$ & $\begin{array}{c}\text { Error } \\
\text { Packets }\end{array}$ & $\begin{array}{c}\text { Lost } \\
\text { Packets }\end{array}$ \\
\hline 18 & 120 & 120 & 0 & 0 \\
\hline 32 & 120 & 120 & 0 & 0 \\
\hline 50 & 120 & 119 & 0 & 1 \\
\hline 70 & 120 & 94 & 17 & 9 \\
\hline
\end{tabular}

The communication range between nodes can be increased by placing the antenna gateway outside the building to reduce the number of obstacles between transmitting and receiving antennas. The parameters of LoRa packets can also be changed to communicate over longer distances. Parameters that can be adjusted include increasing the spreading factor and reducing bandwidth. However, adjusting this parameter increases the packet time on air, thereby increasing the delay between packets.

Data received by the gateway was forwarded to the Thingspeak server. It was written in the appropriate field with the address of the sending node so that it could be accessed via the internet network. Based on the test results, all packets received by the gateway could be forwarded to the Thingspeak server. Figure 10 displays the Thingspeak page.
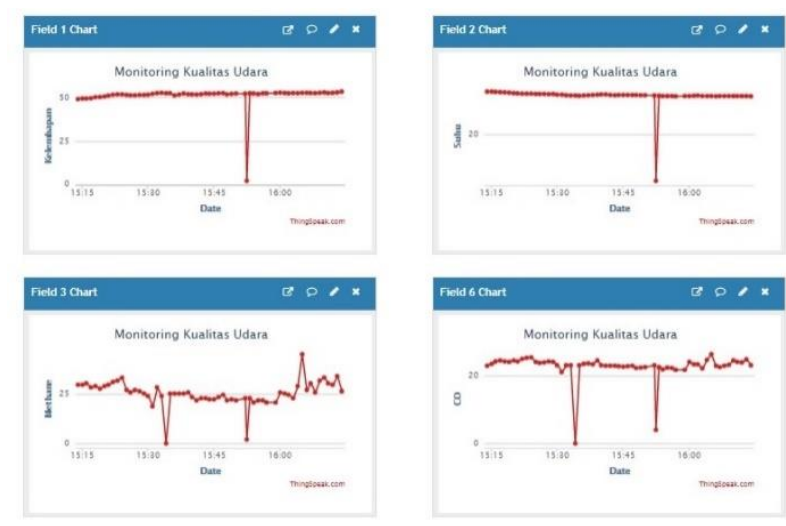

Figure 10 Thingspeak Page

\section{CONCLUSION}

A LoRa-based low cost air quality monitoring system in this study has been able to work well to detect the change of exposure to $\mathrm{CO}, \mathrm{CO} 2$ and PM. It also works well to detect the change of air temperature and humidity. Data from sensor nodes can be sent to the gateway and forwarded to the Thingspeak server. The communication system between sensor node and gateway has been tested with an outdoor-indoor scenario. In testing processes, the sensor node was placed outdoors, and the gateway was indoors. Both devices could communicate up to 32 meters away with $100 \%$ packet received without error. At a distance of 50 meters, 1 out of 120 packets was lost (99.17\% packets received without error). While at a distance of 70 meters, $78.3 \%$ of packets could be received without error.

\section{REFERENCES}

P. Saxena and V. Naik, Eds., Air Pollution Sources, Impacts and Controls. Oxfordshire: CAB International, 2019.

C. Liu et al., "Ambient carbon monoxide and cardiovascular mortality: a nationwide timeseries analysis in 272 cities in China," The Lancet Planetary Health, vol. 2, no. 1, pp. e2-e3, 2018, doi: 10.1016/S2542-5196(17)30181-X.

[3] T. A. Jacobson, J. S. Kler, M. T. Hernke, R. K. Braun, K. C. Meyer, and W. E. Funk, "Direct human health risks of increased atmospheric carbon dioxide," Nature Sustainability, vol. 2, no. 8, pp. 691-701, 2019, doi: 10.1038/s41893019-0323-1.

[4] N. J. Hime, G. B. Marks, and C. T. Cowie, "A comparison of the health effects of ambient PM matter air pollution from five emission sources," International Journal of Environmental Research and Public Health, vol. 15, no. 6, pp. 1-24, 2018, doi: 10.3390/ijerph15061206.

[5] S. Dhingra, R. B. Madda, A. H. Gandomi, R. Patan, and M. Daneshmand, "Internet of things mobile-air pollution monitoring system (IoTMobair)," IEEE Internet of Things Journal, vol. 6, no. 3, pp. 5577-5584, 2019, doi: 10.1109/JIOT.2019.2903821.

[6] H. Gupta, D. Bhardwaj, H. Agrawal, V. A. Tikkiwal, and A. Kumar, "An IoT Based Air Pollution Monitoring System for Smart Cities," 2019 IEEE International Conference on Sustainable Energy Technologies (ICSET), pp. 173-177, 2019.

[7] S. Muthukumar, W. Sherine Mary, S. Jayanthi, R. Kiruthiga, and M. Mahalakshmi, "IoT Based Air Pollution Monitoring and Control System," Proceedings of the International Conference on Inventive Research in Computing Applications, ICIRCA 2018, no. Icirca, pp. 1286-1288, 2018, doi: 10.1109/ICIRCA.2018.8597240.

[8] D. Patil, T. C. Thanuja, and B. C. Melinamath, Air pollution monitoring system using wireless sensor network (WSN), vol. 808. Springer Singapore, 2019. 
[9] S. Kusrey, A. Rai, and V. (Nigam) Saxena, "Zigbee Based Air Pollution Monitoring and Control System using WSN," International Journal of Electronics and Communication Engineering, vol. 4, no. 6, pp. 7-11, 2017, doi: 10.14445/23488549/ijece-v4i6p103.

[10] L. Lin, X. Li, and W. Gu, "PM2.5 monitoring system based on ZigBee wireless sensor network," in 3rd International Conference on Advances in Energy, Environment and Chemical Engineering, 2017, pp. 1-7, doi: 10.1088/17551315/69/1/012094.

[11] I. Kuzminykh, A. Snihurov, and A. Carlsson, "Testing of communication range in ZigBee technology," $2017 \quad$ 14th International Conference The Experience of Designing and Application of CAD Systems in Microelectronics, CADSM 2017 - Proceedings, pp. 133-136, 2017, doi: 10.1109/CADSM.2017.7916102.

[12] M. Rosmiati, M. F. Rizal, F. Susanti, and G. F. Alfisyahrin, "Air pollution monitoring system using LoRa modul as transceiver system,"
Telkomnika (Telecommunication Computing Electronics and Control), vol. 17, no. 2, pp. 586592, 2019, doi: 10.12928/TELKOMNIKA.V17I2.11760.

[13] B. Siregar, H. F. Pratama, and I. Jaya, "LPG leak detection system using MQTT protocol on LoRa communication module," 2020 4th International Conference on Electrical, Telecommunication and Computer Engineering, ELTICOM 2020 Proceedings, pp. 215-218, 2020, doi: 10.1109/ELTICOM50775.2020.9230518.

[14] A. Augustin, J. Yi, T. Clausen, and W. M. Townsley, "A study of Lora: Long range \& low power networks for the internet of things," Sensors (Switzerland), vol. 16, no. 9, pp. 1-18, 2016, doi: 10.3390/s16091466.

[15] U. Noreen, A. Bounceur, and L. Clavier, "A study of LoRa low power and wide area network technology," Proceedings - 3rd International Conference on Advanced Technologies for Signal and Image Processing, ATSIP 2017, 2017, doi: 10.1109/ATSIP.2017.8075570. 